\title{
Les Déterminants Des Conflits De Compétences Dans Le Processus De Décentralisation En Cote d'Ivoire
}

\author{
Guiriobe Paumahoulou Jean-Arsene \\ Enseignant-chercheur, Département d'Anthropologie et de Sociologie \\ Université Alassane Ouattara de Bouaké
}

doi: 10.19044/esj.2016.v12n17p334 URL:http://dx.doi.org/10.19044/esj.2016.v12n17p334

\begin{abstract}
This study identifies and analyses the factors that determine jurisdictional conflicts prevailing in the decentralization process in Côte d'Ivoire. To achieve these objectives, we interviewed, using semi-structured interviews, resource persons in the departments of Alépé, Grand-Bassam, Guiglo and the District of Abidjan. From these interviews, it appears that conflicts of jurisdiction are classified into two categories: vertical conflicts and horizontal conflicts. The vertical conflicts arise between central structures of Government and local authorities. Horizontal conflicts occur between local authorities. Usually caused by structural realities, these conflicts are fueled and poisoned by economic and especially political factors.
\end{abstract}

Keywords: Conflicts of jurisdiction, local authorities, decentralization, central services, factors, oppositions, protest

\section{Resume}

Cette étude identifie et analyse les facteurs qui déterminent les conflits de compétence ayant cours dans le processus de décentralisation en Côte d'Ivoire. Pour atteindre ces objectifs, nous avons interrogé, à l'aide d'entretiens semi-directifs, des personnes ressources dans les départements d'Alépé, Grand-Bassam, Guiglo et le district d'Abidjan. De ces entretiens, il ressort que les conflits de compétences sont classés en deux catégories : les conflits verticaux et les conflits horizontaux. Les conflits verticaux se manifestent entre les structures centrales de l'Etat et les collectivités territoriales. Les conflits horizontaux se produisent entre les collectivités locales. Causés généralement par des faits structurels, ces conflits sont alimentés et envenimés par des facteurs conjoncturels et surtout politiques. 
Mots clés : conflits de compétence, collectivités locales, décentralisation, services centraux, causes, oppositions, manifestations

\section{Introduction}

La décentralisation est un processus dans lequel l'Etat se dessaisit, au profit des collectivités territoriales dont l'administration est assurée par des assemblées élues et disposant d’une liberté de décision, des compétences exercées jusque-là par ses organes centraux et ses représentants territoriaux (Secrétariat d'Etat français à la coopération, 1997). La répartition des compétences entre les collectivités territoriales et l'État s'effectue de telle sorte que chaque domaine de compétences ainsi que les ressources correspondantes soient affectés en totalité soit à l’État, soit aux communes, soit aux départements, soit aux régions. Pour ce faire, l’on distingue, dans la mesure du possible, celles qui sont en effet mises à la charge de l'État et celles qui sont dévolues aux communes, aux départements ou aux régions (Lefèvre, 2011). Il s’agit donc d'une répartition verticale et horizontale de domaines de compétence. La verticalité concerne la répartition de compétences entre les collectivités locales et les organes centraux de l'Etat. Quant à la répartition horizontale, elle a lieu entre les différentes catégories de collectivités locales exerçant sur toute l'étendue du territoire national. Ainsi, l'Etat, les services centraux et déconcentrés des ministères concernés par les compétences transférées aux collectivités, de même que les agences et établissements publics nationaux deviennent-ils des partenaires privilégiés de celles-ci, dans ce processus (Ministère sénégalais de l'aménagement du territoire et des collectivités locales, 2011). Mais, malgré toutes ces dispositions réglementaires, l'on constate très souvent la manifestation de conflits opposant ces différents acteurs dans l'exercice pratique de ces compétences. Ces conflits engendrent la suspension ou l'arrêt complet des travaux, le retard dans le déroulement des activités ou l'abandon pur et simple de celles-ci et surtout des pertes de temps considérables avant que la décision de l'arbitre ne tombe. En effet, un nombre impressionnant de projets d'investissements est mis en veilleuse à cause de ces conflits, en attendant l'évacuation des incompréhensions (Guiriobé, 2012). Ils mettent donc en mal le processus de décentralisation et de développement local.

Ces effets ont sans doute poussé certains auteurs à mener des études sur ce genre de conflits afin de cerner leurs contours pour pouvoir les résoudre. Mais, la plupart d'entre eux se sont focalisés sur l'analyse de la manifestation, la typologie et les différentes méthodes de gestion et de prévention de ces conflictualités (Hond, 2014 ; Lefèvre, 2011 ; Diop et Badara, 2013).

Peu d'études semblent avoir été consacrées à l'identification et à l'analyse des facteurs qui déterminent ces conflits. Et pourtant, une 
meilleure connaissance des facteurs à l'origine de ceux-ci est utile dans la mesure où cette connaissance peut favoriser leur neutralisation et par conséquent leur réduction. Ainsi, le présent article se propose-t-il d’identifier et d'analyser, à travers le cas précis des départements d’Alépé, GrandBassam, Guiglo et du district d'Abidjan en Côte d'Ivoire, les éventuelles causes des conflits de compétence se manifestant dans le processus de décentralisation en cours dans ce pays. Autrement dit, quels sont les différents facteurs qui sont à l'origine des conflits de compétences qui surviennent au niveau du processus de décentralisation dans les départements d’Alépé, Grand-Bassam, Guiglo et du district d’Abidjan en Côte d’Ivoire ?

\section{Materiels et methodes}

Cette étude, effectuée en 2010, a pour objectif d’identifier et d'étudier, dans une perspective de cas, les facteurs qui déterminent les conflits de compétences qui se manifestent au niveau du processus de décentralisation en Côte d’Ivoire. Les départements d’Alépé, Grand-Bassam de Guiglo et le district d'Abidjan ont été choisis pour deux raisons essentielles. Dans les départements d’Alépé et de Guiglo, les autorités locales des conseils généraux et celles des municipalités sont toutes issues d'une même organisation politique, alors qu'au niveau du département de Grand-Bassam et du district d'Abidjan, les collectivités locales, toute catégorie confondue, sont dirigées par des élus locaux issus de différents partis politiques. Selon les services contentieux du district d'Abidjan, les conflictualités deviennent manifestes lorsque les élus des collectivités en conflit ont des opinions ou préférences politiques opposées. Aussi, en ce qui concerne la zone départementale d'Abidjan plus particulièrement, plusieurs conflits de compétences ont opposé le district à de nombreuses communes de la ville d'Abidjan. Ainsi, au cours de l'enquête qui s'est déroulée sur une période d'un mois, c'est-à-dire août 2010, avons-nous, par le biais d'entretiens directs et compréhensifs, cherché à questionner l'existence de conflits de compétences, leurs différentes modalités, leurs manifestations, les objets de contestation et les divers modes de résolution de ces conflits. Les entretiens ont été menés auprès de 40 personnes au profil pertinent, c'est-àdire 08 enquêtés par départ (élus locaux, fonctionnaires détachés auprès des collectivités visitées), 04 agents des structures étatiques ayant été opposées à des entités décentralisées lors de conflits de compétences et 04 autres de la Direction Générale de la Décentralisation et du Développement Local. Ces entretiens et l'observation directe du déroulement de certaines conflictualités ont permis d'apprécier, les circonstances qui entourent les conflits de compétences qui se sont manifestés entre ces collectivités territoriales. Avec ces interviews, nous avons pu réunir plusieurs témoignages qui ont permis 
d’identifier et de comprendre en profondeur les différents déterminants ou facteurs de ces conflits.

Les informations collectées ont été analysées à l’aide de la théorie de la justification car les acteurs des conflits de compétence analysés dans le cadre de cette étude se réfèrent à des principes ou encore, à une forme de biens communs : on ne revendique pas seulement parce que l'on désire, mais au nom d'une conception de ce qui est juste dans cette circonstance. Autrement dit, on ne s'affronte pas seulement pour soi : on revendique au nom d'une valeur générale, publiquement revendiquée face aux autres acteurs. Il s’agit là de principes supérieurs communs, c'est-à-dire des principes politiques et économiques (Jacquemain, 2001).

Cette approche permet d'aborder la question de l'identification et de l'analyse des déterminants des conflits de compétences qui se manifestent au niveau du processus de décentralisation dans les départements visités, à travers trois points focaux. Après avoir montré les différentes typologies et les manifestations des conflits de compétences, nous présenterons ensuite les déterminants structurels et, enfin les facteurs conjoncturels et politiques.

\section{Resultats de la recherche}

\section{Typologie et manifestation des conflits de compétences.}

La compétence est en fait l'aptitude d'une autorité administrative ou judiciaire à procéder à certains actes dans des conditions déterminées par la loi. Le conflit de compétence, quant à lui, est un conflit susceptible de surgir dans un Etat fédéral lorsqu'un pouvoir excède ses compétences en adoptant une législation. Le plus souvent, il y a conflit de compétence lorsqu'une des entités de l'Etat fédéral (Autorité fédérale, communauté ou région) affirme qu'une loi, un décret ou une ordonnance émanant d'une autre entité excède la compétence de celle-ci. Il est question donc d'un conflit entre deux pouvoirs possédant une capacité législative. En d'autres termes, il s’agit de conflits qui se produisent lorsque, dans la mise en œuvre de sa politique, l'autorité fédérale, une communauté, un département ou une région ne respecte pas les règles prescrites en matière de répartition des compétences.

Cette répartition des compétences a été en effet faite en Côte d’Ivoire sans la consultation préalable des collectivités territoriales et sans aucune confrontation entre les différents destinataires, comme l'a fait remarquer le deuxième vice-président du conseil général d’Alépé: «le séminaire consacré à la répartition des compétences n'a pas enregistré la présence des collectivités locales ou de leurs représentants ». Dans ce même ordre d’idées, il ajoute ceci : "L'exercice pratique de ces responsabilités ainsi délimitées et transférées, génère des conflits situés à deux niveaux différents, à savoir les conflits opposant les structures centrales de l'Etat aux collectivités locales, c'est-à-dire les conflits verticaux et ceux qui se 
manifestent entre les collectivités territoriales, c'est-à-dire les conflits horizontaux ».

Au niveau des conflits verticaux, les Ministères et EPN, en tant que services centraux de l'Etat, détiennent des compétences considérables dont certaines parties ont été transférées aux structures décentralisées. Ils ont désormais pour missions de réglementer, définir les normes et procédures transversales, de suivre et de contrôler leur application, superviser, coordonner, mais aussi de réaliser des projets, travaux et équipements d'envergure nationale qui structurent le territoire et constituent par conséquent les fondements de son aménagement. Mais, selon les agents interrogés à la Direction Générale de la Décentralisation et du Développement Local (DGDDL), la réalité du terrain est plus que préoccupante car des ministères ont été, à plusieurs reprises, opposés aux collectivités locales : "Il existe plusieurs points d'achoppement entre certains ministères et les collectivités dans divers domaines. D'autres ministères, du fait de leur accointance dans la gestion des biens domaniaux de l'Etat avec les collectivités, se retrouvent très fréquemment opposés à celles-ci plus que les autres » (Agent T. S.) ; "Le ministère des villes et de la salubrité urbaine, à travers la gestion des ordures ménagères et celle de la salubrité urbaine à Abidjan, a été, à plusieurs reprises, confronté au district d'Abidjan et aux communes composant ce district. Le ministère de l'éducation nationale a continué de pourvoir en équipements tels que les tables-bancs, les manuels scolaires et autres aux établissements scolaires, alors qu'il a pris les dispositions légales pour que ces activités soient faites par les conseils généraux. Quant au ministère de la santé et de l'hygiène publique, il a continué de fournir les hôpitaux et autres établissements sanitaires en médicaments. Pourtant, cette activité ne fait plus partie de ses responsabilités car il a signé les arrêtés devant permettre aux conseils généraux de l'exercer à sa place. Ce sont en fait ces agissements qui ont fait naître des situations conflictuelles entre ces ministères et les collectivités locales » (Agent D. D.). L’ on ne saurait cependant énumérer les différentes modalités des conflits se manifestant dans ce processus de décentralisation sans faire mention de ceux opposant les collectivités territoriales aux établissements publics nationaux.

Les conflits qui ont opposé en effet les établissements publics nationaux aux collectivités locales sont tout aussi nombreux, comme le précise le secrétaire général du district d’Abidjan : «Il y a plusieurs points d'achoppements entre les EPN et les collectivités territoriales ». Il a cependant cité un cas particulier qui a mis en conflit toutes les catégories de collectivités locales à un établissement public national dénommé A.G.T.U. (Agence de Transports Urbains) : "Le noud du conflit opposant l'A.G.T.U à l'ensemble des collectivités territoriales porte sur la délivrance des 
autorisations de transport urbain ». En effet, l’A.G.T.U. ne peut pas délivrer des autorisations de transport urbain, comme le soutient le premier vicegouverneur du district d’Abidjan: «L'AGTU n'a pas la compétence de délivrer les autorisations de transport urbain, parce que d'une part, cette activité ne faisait plus partie des missions de régulation de cette structure tel que prévu par l'article 32 de l'ordonnance n 2000 - 67 du 09 Février 2000, portant définition des principes fondamentaux du régime des transports terrestres mais, d'autre part, cette responsabilité a été dévolue aux collectivités locales par les lois sur la décentralisation, notamment la loi $n^{\circ}$ 2003 - 208 du 07 juillet 2003, portant répartition et transfert des compétences de l'Etat aux collectivités, en ses articles 10 ; 15 et 21 et la loi $n^{\circ} 2003$ - 489 du 26 décembre 2003, portant régime financier, fiscal et domanial des collectivités locales, en ses articles 134 ; 145 et suivant et 159 ». Les agents de l'A.G.T.U. s’appuient, quant à eux, sur d'autres textes juridiques pour contredire les propos des élus locaux. C'est dans ce cadre que s'inscrit l'agent Z. B. lorsqu'il avance ceci : «il faut s'en tenir à l'application des textes de l'article 35 de la loi $n^{\circ} 2004$ - 271 du 15 avril 2004 portant loi de finances de l'année 2004 qui donne compétence exclusive à l'A.G.T.U en matière de recouvrement de redevances et d'autorisations ».

En ce qui concerne les conflits horizontaux, ils opposent les conseils généraux et les communes et/ou les districts et les communes. Cette situation découle, de la cohabitation sur une même circonscription départementale, des conseils généraux ou districts avec des communes, comme le dit le premier vice-gouverneur du district d'Abidjan: «La cohabitation des conseils généraux et des communes sur les mêmes circonscriptions départementales va engendrer de nombreux conflits dont l'envergure dépendra de l'objet de discorde et de l'appartenance politique des élus locaux en conflit. Il s'agit exactement de conflits qui opposent les conseils généraux et les communes avec lesquelles ils partagent le même territoire départemental et/ou les districts et les communes de leur ressort territorial ».

Les conflits de compétences qui ont eu lieu en effet entre les conseils généraux et les communes avec lesquelles ils partagent le même territoire départemental se sont manifestés, dans la plupart des cas, dans le domaine fiscal, la gestion des ordures ménagères et l'entretien de la voierie. C'est ce que déclarent le secrétaire général du conseil général de Guiglo et le deuxième adjoint au maire de la municipalité de Grand-Bassam : " c'est la perception de certaines taxes routières et redevances qui a opposé, à plusieurs reprises, la municipalité et le conseil général de cette localité. Ces taxes, qui revenaient autrefois aux communes, ont été réattribuées aux conseils généraux qui sont de nouvelles collectivités locales » (Secrétaire 
général du conseil général de Guiglo) ; «La perception des taxes routières et redevances constituent, dans la plupart des cas, l'objet de conflits entre la mairie et le conseil général » (Deuxième adjoint au maire de la municipalité de Grand-Bassam). Au niveau du district et des communes d’Abidjan, les conflits ont porté principalement sur la gestion des ordures ménagères, l'entretien de la voirie, la construction et l'urbanisme, d'après un agent des services contentieux du district d'Abidjan qui a déclaré ceci : «La commune de Treichville qui prévoyait réaliser un projet de construction d'un centre commercial sur l'espace du rond point de l'avenue 8, rue 12, a vu se dresser contre elle le district, au motif que l'espace en question fait partie de son patrimoine. La teneur de ce conflit s'apprécie à la lumière des courriers que se sont adressées les autorités locales. Ayant en fait constaté le début des travaux réalisés par les services techniques de la mairie de Treichville, le gouverneur du district d'Abidjan a été le premier à adresser un courrier au maire de Treichville afin de l'arrestation des travaux ». L'espace du rond point de la commune de Treichville a été ainsi le théâtre de la contradiction de deux intérêts appartenant à deux personnes morales distinctes partageant le même territoire et les matières sont nombreuses dans ce cas. Le Ministère de tutelle, c'est-à-dire le Ministère de l'intérieur et de la décentralisation a donné, lors de son arbitrage, raison au district au détriment de la commune de Treichville.

Il faut cependant noter que l'intensité ou l'ampleur des conflits de compétence qui se sont déroulés entre les collectivités locales diffère d'une localité à une autre. Cette différence dépend, selon les secrétaires généraux des collectivités visitées et les agents interrogés à la DGDDL, de l'appartenance politique des élus locaux : "Si les autorités des collectivités en litige sont issues d'un même parti politique, le conflit est sans incident et reste méconnu du public car les élus locaux s'arrangent à trouver un compromis sans avoir recours à l'arbitrage de l'autorité de tutelle » (Agent P. L.) ; "Les conflits restent à l'état latent dans les départements où toutes les collectivités, c'est-à-dire les municipalités et les conseils généraux sont dirigés par des élus issus d'un même parti politique car ceux-ci laissent faire et les situations conflictuelles finissent toujours par s'arranger d'ellesmêmes » (Secrétaire général de la mairie de Guiglo). Cette situation ainsi dépeinte concerne les départements de Guiglo et d'Alépé où les élus locaux sont tous du même bord politique. Ce qui n'est pas du tout le cas dans les deux autres zones d'étude, à savoir Abidjan et Grand-Bassam où les autorités des collectivités territoriales choisies pour l'enquête de terrain appartiennent à des partis politiques qui s'affrontent pour le contrôle et l'exercice du pouvoir de l'Etat central. Les conflictualités entre ces collectivités se manifestent, d'après les agents de la DGDDL, au grand jour et engendrent par la même occasion des incidents majeurs : " Le district d'Abidjan a eu 
recours à la gendarmerie pour pouvoir démolir les travaux effectués par la mairie de Treichville» (Agent P. L.) ; «Dans le département de GrandBassam, les conflits de compétence entre le conseil général et la mairie de Grand-Bassam occasionnent des divisions au sein de la population en fonction des clivages politiques » (Agent D.D.).

Ces conflits de compétences, classés en conflits verticaux et horizontaux, font remarquer que le processus de décentralisation, dans les départements visités dans le cadre de cette étude, constitue, avec la multiplicité des autorités et la superposition des territoires des circonscriptions administratives et des institutions décentralisées qui le caractérisent, un terrain incommensurablement propice aux tensions conflictuelles. Pour mieux apprécier ces conflits et par conséquent leurs enjeux, il convient d'en rechercher les facteurs qui les déterminent.

\section{Les facteurs structurels des conflits de compétences}

Les conflits de compétences ci-dessus énumérés sont occasionnés par une diversité de facteurs parmi lesquelles l'on peut citer les facteurs structurels, comme le souligne le secrétaire général du conseil général de Grand-Bassam: "Les conflits de compétence qui se manifestent dans le processus de décentralisation sont pour la plupart entraînés par plusieurs facteurs dont les plus importants sont d'ordre structurel. Il s'agit exactement de l'imprécision et des diverses interprétations des textes ».

Par imprécision des textes, il faut entendre à la fois les problèmes soulevés par la succession des textes dans le temps, par l'unicité de leur objet et par la pluralité de leurs destinataires. Lorsque des textes portant sur un même objet interviennent à des époques différentes, il se pose la question de savoir lequel d'entre eux peut l'emporter sur les autres? La question est résolue différemment.

D’abord, au nom du principe de la hiérarchie des normes, il est donné une autorité supérieure à la loi qui de, ce fait, est au dessus des règlements et même des ordonnances avant leur ratification. Ensuite, si des textes, se suivant dans le temps et portant sur un même objet, jouissent de la même autorité, c'est un autre principe qui règle le conflit qui peut naître entre ces textes. C'est le principe de « la règle spéciale déroge à la règle générale ». Si les textes en conflit sont deux lois spéciales, c'est la plus récente qui l'emporte eu égard au principe général: "la règle postérieure déroge à la règle antérieure’'. Mais, qu'en est-il, dans le cas où une loi générale est postérieure à une loi spéciale, les deux, bien-entendu, étant relatives au même objet ? La solution est plus délicate. Il faut alors rechercher l'intention du législateur qui a pu vouloir laisser subsister la loi ancienne dans son domaine particulier au lieu de l'abroger. Cependant, en règle générale, on 
considère que la loi ancienne subsiste comme loi spéciale avec, bien sûr, les conséquences qui s’y attachent.

Les quelques principes juridiques énoncés ci-dessus sont sans intérêt lorsque les différents textes en conflit sont stipulés au profit d'un seul destinataire. Il en va différemment quand ces lois mettent en concurrence plus d'un destinataire, et c'est là que le conflit de compétence trouve tout son sens, comme cela a été le cas ci-dessus cité, c'est-à-dire l’objet des différents conflits entre l'AGTU et les collectivités locales. Un autre exemple spécifique est celui de la loi de 2003, relative au transfert et répartition de compétences en matière de voies de communication et des réseaux divers. Les conseils généraux et les communes ont reçu exactement des compétences pour «la création, la gestion et l'entretien des voies de communication et des réseaux divers ainsi que les pistes rurales ». Lorsque deux collectivités de nature différente se retrouvent avec les mêmes compétences, les spécifications sont faites par des décrets d’application. Mais, pour ce qui est de cette loi précitée, le décret de son application est, jusqu'au jour de l'enquête, attendu par les concernés. Il en résulte un grand nombre de conflits entre ces collectivités locales. Une situation qui a donc poussé le gouvernement à opter pour des textes généraux, ce qui permet d'éviter les conflits et la pluralité des textes, en harmonisant le statut de toutes les collectivités territoriales (département, districts et communes). Mais, cet état de fait est de nature à augmenter, malgré tout, les conflits car, " chaque collectivité est dans la logique d'interpréter le texte au gré de ses intérêts » (Secrétaire général de la mairie de Grand-Bassam).

En effet, un texte, même apparemment clair n’échappe pas toujours aux interprétations des destinataires qui, volontiers, veulent lui donner un sens correspondant ou du moins un sens prenant en compte leurs opinions. Il n'est pas étonnant, de ce fait, de voir des conflits basés sur des interprétations témoignant souvent de l'incompréhension de ces textes, en raison de leurs caractères très souvent ambigus et flous. Selon le deuxième vice-président du conseil général d’Alépé, "des conflits sont ainsi nés autour des prélèvements de certaines taxes par les conseils généraux et les communes qui composent un même département. Certains prélèvements de taxes surtout les taxes routières qui revenaient, bien avant la création des conseils généraux, aux communes, sont en fait passées aux mains des conseils généraux dès leur instauration ». Le problème ici est, que les communes continuent ou veulent continuer, dans la plupart des cas, de percevoir ces taxes en lieu et place des conseils généraux car, pour les autorités municipales, "ces taxes portent toujours, dans les textes réglementaires, la mention "commune" » (Premier adjoint au maire de la mairie de Guiglo). Selon les élus départementaux «ces taxes reviennent pourtant aux départements d'après la nouvelle loi portant transfert et 
répartition des compétences de l'Etat aux collectivités locales » (Président du conseil général de Guiglo).

Au regard de ce qui précède, l’on retiendra que les conflits de compétences qui se sont manifestés entre les collectivités territoriales des départements visités découlent de l'imprécision et de la succession des textes dans le temps et surtout de l'interprétation de ceux-ci. Ils proviennent donc de la situation des textes réglementant le cadre juridique et institutionnel de la décentralisation, c'est-à-dire de l'organisation structurelle du processus. Mais, à côté de ces causes évidentes, existent d'autres qui ne peuvent être négligées car leur prise en compte est aussi importante pour cette présente étude et surtout pour la gestion et la prévention de ces conflits.

\section{Les facteurs conjoncturels et politiques}

Selon le directeur des services financiers du conseil général de Guiglo, "c'est en effet le contrôle des flux financiers qui accompagnent les compétences qui engendre des conflictualités entre les différents acteurs impliqués dans le processus. L'imprécision et les interprétations des textes réglementaires ne sont que des prétextes ». Le chef comptable de la mairie de Grand-Bassam ne dit rien d'autre lorsqu'il avance ceci : " les diverses interprétations des textes ne sont que l'émanation de désirs ardents de conserver ou d'obtenir des ressources financières car une compétence n'est pas seulement une aptitude de procéder à un acte, mais aussi et surtout une source de revenu pour la collectivité locale en question. Dans ce cas précis, perdre ou laisser échapper une compétence au profit d'une autre structure n'est pas chose aisée car cela diminue considérablement le budget. Mais, l'acquisition d'une nouvelle compétence est plutôt considérée comme celle de ressources financières supplémentaires pour la municipalité ». Ce qui lui fait dire enfin de compte que «la compétence est d'abord et surtout une source de revenu pour la collectivité ». Ce point de vue est partagé par le chef comptable de la mairie d’Alépé qui s’est demandé « si les collectivités locales peuvent se plaindre si on leur retranche une compétence sans toucher aux ressources financières qui l'accompagnent ». Mais, il répond par la négation car pour lui, «ce sont les ressources financières qui comptent car aucune collectivité locale ne cherchera à s'opposer à une autre si celleci exerce une compétence qui lui est dévolue sans toucher aux ressources financières qui accompagnent son exercice ».

Les fonctionnaires détachés auprès des collectivités locales visitées ont plutôt pointé du doigt les raisons politiques comme étant à l'origine des conflits de compétence qui opposent les différentes collectivités territoriales visitées : "Les véritables causes des conflits de compétence qui opposent les structures décentralisées sont d'ordre politique car les élus locaux ne sont que des représentants locaux des groupements politiques les plus importants 
du pays, c'est-à-dire le FPI et le RHDP. A ce titre, ils constituent de puissants relais politiques dans leur différente collectivité » (Le Directeur du développement humain du conseil général d’Alépé). Ainsi, les situations conflictuelles relatives à l'exercice de certaines compétences deviennentelles le prolongement de la lutte politique pour l'exercice ou la conquête du pouvoir d'Etat. "C'est pourquoi, les conflictualités sont plus manifestes, avec des incidents majeurs lorsque les collectivités locales en conflit sont dirigées par des autorités issues de partis politiques opposés sur la scène nationale, comme c'est le cas à Grand-Bassam où la municipalité est aux mains du RHDP et le conseil général aux mains du FPI », (Chef personnel de la municipalité de Grand-Bassam). A ce sujet, certains agents interrogés à la DGDDL ont plutôt cité le cas du conflit ayant opposé le district d'Abidjan (FPI) et la commune de Treichville (RHDP).

En un mot, nous dirons que, pour certains fonctionnaires détachés auprès des collectivités visitées, les causes profondes des conflits de compétence qui se manifestent très souvent entre les structures impliquées dans le processus de décentralisation et de développement local sont d'ordre économique et, pour d'autres, elles sont d'ordre politique, la situation des textes n'étant qu'un prétexte.

\section{Analyse et discussion}

Les résultats ont révélé l'existence de conflits verticaux et horizontaux dont les déterminants sont d'ordre structurel et surtout conjoncturel et politique.

La décentralisation désigne en effet un transfert de compétences de l'État à des collectivités territoriales, dont les organes dirigeants sont élus. Elle s'accompagne souvent, mais pas toujours, du transfert de différentes ressources censées leur permettre de faire face à l'augmentation des dépenses qu'implique l'exercice de ces compétences (Le Lidec, 2010). Elle se définit également comme le résultat d'un effort institutionnel global entrepris par un Etat et visant à transférer des pouvoirs de décisions à des collectivités territoriales en vue d'organiser le développement local (Maetz et Quieti, 1987). Par cette décentralisation, l'Etat ivoirien a donc transféré aux collectivités territoriales une partie de ses compétences. Aucune n’exerce de tutelle sur les autres et elles doivent collaborer, leurs compétences se complétant dans plusieurs domaines. La répartition des compétences entre les collectivités territoriales et l'État s'est en fait effectuée, dans la mesure du possible, en distinguant celles qui sont mises à la charge de l'État et celles qui sont dévolues aux communes, aux départements et aux districts, de telle sorte que chaque domaine de compétences ainsi que les ressources correspondantes soient affectés en totalité soit aux services centraux et déconcentrés de l'État, soit aux communes, soit aux départements, soit aux 
districts (Lefèvre, op.cit.). La répartition des domaines de compétences et des ressources correspondantes a donc été faite d'une manière verticale et horizontale, c'est-à-dire entre les services centraux de l'Etat et les structures décentralisées d'une part et, entre les structures décentralisées d'autre part. Mais, dans la pratique, la délégation de pouvoir dans les domaines de compétences transférés aux collectivités et à leurs organes élus a conduit à bien des égards, à une recrudescence de conflits de compétences (Diop et Badara, 2013). Ces antagonismes surviennent quant des décisions ne peuvent être prises par les procédures habituelles d'un processus de décentralisation (Rui, 2011). Ils font partie intégrante des fléaux qui contribuent au retard de l’éclosion des communes, départements et régions (Evina, 2015).

Ainsi, le processus de décentralisation en Côte d'Ivoire est-il en proie à de nombreux conflits de compétences qui se manifestent à deux niveaux. Ils opposent d'une part les collectivités locales se partageant la même circonscription départementale, c’est-à-dire les conseils généraux et les communes de leur ressort territorial ou le district et les municipalités qui composent la ville d'Abidjan (conflits horizontaux) et, d'autre part certaines structures centrales de l'Etat à ces collectivités (conflits verticaux). Les conflits verticaux opposent en effet les structures centrales de l'Etat tels que les ministères et les EPN (Etablissements Publics Nationaux) aux collectivités locales et les conflits horizontaux décrivent les antagonismes qui opposent une collectivité locale à une autre. Ces résultats sont quelque peu identiques à ceux obtenus au Cameroun (Hond, 2014). La seule différence est que, dans le cas ivoirien ou de cette présente étude, l'Etat, en tant que collectivité mère, ne s'est pas trouvée dans une situation conflictuelle avec les collectivités locales concernée par cette enquête. Ce sont plutôt certaines de ses structures centrales et de ses administrations déconcentrées telles que les préfectures et les sous-préfectures qui se sont retrouvées dans des situations conflictuelles avec les structures décentralisées.

Aussi, l'étude a-t-elle fait ressortir que ces conflits de compétence ainsi catégorisés sont engendrés par plusieurs facteurs tels que les facteurs structurels, conjoncturels et politiques. Ces observations sont différentes de celles qui ont été faites en France (Rui, op.cit.), au Cameroun (Hond, op.cit.) et au Sénégal (Diop et Badara, op.cit.). Cette différence vient du fait que dans le cas français, les antagonismes surviennent quant des décisions ne peuvent être prises par les procédures habituelles d'un processus de décentralisation. Au Cameroun, ces conflits naissent plutôt du déséquilibre en termes de prérogatives entre le pouvoir central et les entités locales dans l'organisation distributive des compétences locales par l'Etat et dans la formalisation des modalités de gestion de ces compétences. Alors qu'au 
Sénégal, ils sont engendrés par la fragmentation du territoire avec la création de communes d'arrondissement à partir de 1996.

En effet, les facteurs structurels des conflits de compétence observés dans les départements d’Alépé, Grand-Bassam, Guiglo et le district d'Abidjan découlent de l'organisation ou des principes organisationnels et institutionnels du processus de décentralisation, c'est-à-dire de l'imprécision de certains des textes réglementant le cadre juridique et institutionnel dans lequel évoluent et exercent les collectivités locales. Cette imprécision occasionne des interprétations au gré des intérêts. Ces causes structurelles ont été surtout mises en exergue par les élus locaux. Ils l'ont fait afin de dissimuler les déterminants conjoncturels et surtout politiques que les fonctionnaires ou les agents de l'Etat central détachés auprès des collectivités territoriales visitées ont pointé du doigt comme étant les véritables facteurs qui sont à l'origine de ces conflits.

Les conflits de compétences qui se sont en effet manifestés au niveau du processus de décentralisation dans ces départements ont certes des origines structurelles, mais aussi et surtout des causes conjoncturelles et politiques. Ceux qui ont opposé les administrations centrales et déconcentrées de l'Etat aux collectivités territoriales sont alimentés par des facteurs structurels et économiques et ceux qui se sont manifestés entre les collectivités locales sont le fruit de facteurs structurels, économiques et/ou politiques. Les collectivités locales sont en effet dirigées pour la plupart par des élus locaux issus de différentes forces politiques du pays. Ces derniers sont donc des représentants locaux de leur parti politique dans leur commune ou département. Ainsi, lorsque des collectivités locales se partageant un même territoire départemental sont-elles dirigées par des autorités issues de différentes organisations politiques, les conflits de compétence, ayant certes des causes structurelles, sont plutôt alimentés et envenimés par des principes politiques. C'est la raison pour laquelle ils se manifestent, dans ce cas, au grand jour, avec des incidents majeurs et, dans la recherche de leur résolution, les collectivités en conflit se remettent à l'arbitrage de l'autorité de tutelle qui utilise parfois la force ou la contrainte pour faire passer ses décisions. Cela a été le cas entre le district d'Abidjan, dirigé par le FPI et la municipalité de Treichville, dirigée par le RHDP, ainsi que le conseil général de Grand-Bassam (FPI) et la municipalité de Grand-Bassam (RHDP). Par contre, dans les départements d'Alépé et Guiglo, les conflits de compétence qui ont opposé les conseils généraux, dirigés par le FPI et les municipalités, également aux mains du FPI, sont restés à l'état latent et n’ont jamais été portés devant l'autorité de tutelle. Il n’y a pas eu d'échanges de courriers, ni d'échanges ou d'affrontements verbaux entre les élus locaux afin de ne pas occasionner la désagrégation du parti dans ces dits départements car, ayant la même coloration politique, ils obéissent aux mêmes principes, partagent une 
vision commune et des biens communs sur le plan politique (Jacquemain, op.cit.). Cette situation découle de la lutte entre les deux formations politiques (FPI et RHDP) pour l'exercice et/ou la conquête du pouvoir de l'Etat central.

\section{Conclusion}

Les conflits de compétences se produisent lorsque, dans la mise en œuvre de sa politique, l'autorité centrale, une communauté, un département ou une région ne respecte pas les règles prescrites en matière de répartition des compétences dans un processus de décentralisation. Ceux qui ont eu lieu dans les départements d'Alépé, Grand-Bassam, Guiglo et le district d'Abidjan en Côte d'Ivoire, sont classés en deux catégories : les conflits verticaux qui opposent les services centraux et déconcentrés de l'Etat aux collectivités locales et les conflits horizontaux se manifestant entre les collectivités locales. Toutes ces deux catégories de conflits sont engendrée par des facteurs structurels et surtout conjoncturels et/ou politiques. Cependant, les facteurs structurels, malgré leur généralité et leur évidence en la matière, ne servent que de prétexte aux parties en conflit car les oppositions sont plutôt motivées et envenimées par des raisons économiques et surtout politiques. Dans un tel contexte, une réduction ou une neutralisation de ces aspects, qui passe par une étude approfondie sur les méthodes de gestion et de prévention de ce type de conflits, est donc nécessaire dans ce processus de décentralisation afin d'éviter ou de réduire, dans un futur proche et lointain, ces situations conflictuelles.

\section{References:}

Diop D. et Sy B. A., 2013, Acte 3 de la décentralisation au Sénégal : le point de vue des urbanistes, Dakar, consulté sur internet : file : /// : / User / ACER / Documents / Acte 3 de la décentralisation au Sénégal_le point de vue des urbanistes. Htm, 03 pages.

Evina N. J. F., 2015, Gouvernance territoriale: enjeux et paradoxes, CIDEGEF, Douala (Cameroun), consulté sur internet: http : // WWW. Cidegef-refer-org / douala / NGOK EVINA doc. 10 pages.

Guiriobé P. .J. A.., (2012). Décentralisation et développement endogène en Côte d'Ivoire, Thèse, IES, Université Félix Houphouët Boigny, Abidjan, 431 pages.

Hond T. J., 2014, «La prévention et la résolution des conflits dans un système décentralisé : l'expérience du Cameroun ", In Rapport du séminaire sur les stratégies et politiques innovantes de décentralisation dans les pays africains, 27-29 janvier 2014, CAFRAD / FHS / ACBF, Tanger (Maroc), consulté sur internet : http : // WWW. cafrad. org. / Workshops / Tanger 2729_01_14 / Dr_Hond_document_2. Pdf, pp 3-5. 
Jacquemain M., 2001, Les cités et les mondes : le modèle de la justification chez Boltanski et Thevenot, Département de sciences sociales, Université de Liège, consulté sur internet : https: // orbi. ac. be / bibstream / 2268 / 90443 /// Les \% 20 cités-et-mondes, 28 pages.

Lefèvre A., 2011, "Clarification de la répartition des compétences entre l'Etat et les collectivités territoriales ", Rapport d'information $n^{\circ} 283$, Présidence du Senat français, 15 pages.

Le Lidec P., 2010, « Les relations financières entre l’Etat et les collectivités territoriales: un sauvetage des conseils généraux orchestrés au prix », In Informations sociales $n^{\circ} 162$, CNAF, Paris, consulté sur internet : http : // WWW. caim .info // revue-informations-sociales-2010-6-page-32. htm, pp 32-40.

Maetz, M. et Quieti, M., 1987, Formation pour la planification décentralisée : leçons tirées de l'expérience, Rome, FAO, 59 p.

Ministère sénégalais de l'aménagement du territoire et des collectivités locales, 2011, «Clarification des rôles et responsabilités des acteurs de la décentralisation », Rapport final, Dakar.

Rui S., 2012, "Conflit ", In les 100 mots de la sociologie, PUF, Que saisje ?, Paris, consulté sur internet : URL : http : // Sociologie. revues. org / 676, pp 54-55.

Secrétariat d'Etat français à la coopération, 1997, La décentralisation en Afrique subsaharienne, Brunnetfrançoise Ed, Paris. 